\title{
Construcción de la magnitud área en estudiantes de grado $5^{\circ}$
}

\section{Construction of the scale area 5th grade students}

Construção do Espaço alunos do 5 escala

Recibido: mayo de 2013

Aprobado: agosto de 2013
Geral Stivens Galán García ${ }^{2}$

Yeimy Rodríguez García ${ }^{3}$

\section{Resumen}

La propuesta didáctica se centra en la enseñanza-aprendizaje de la magnitud área en el grado quinto de educación básica primaria. Se diseña e implementa una secuencia de actividades bajo la teoría de situaciones didácticas propuesta por Brousseau; y se estructura según las fases para la construcción de la magnitud planteadas por Chamorro y Belmonte, las cuales se ponen en correspondencia con los estadios Piagetianos sobre el desarrollo de la medida de lo cual se obtiene una caracterización más detallada para construir una magnitud perceptible cualquiera con su respectiva unidad de medida de manera progresiva, a lo cual se incorporan los conocimientos didácticos de Rosa Corberán, que enfatizan en el tratamiento sobre la magnitud área y se ajustan a las necesidades del estudiantado.

Considerando la relación de dicha Actividad Pedagógica del maestro de matemáticas con su ideario pedagógico y la necesidad de organización de la enseñanza, propongo por medio del trabajo con los maestros una posibilidad para llevar al aula de clase los elementos construidos culturalmente.

Palabras clave: Matemáticas escolares, medida, magnitudes, área, diseño, gestión y evaluación, propuesta didáctica.

\begin{abstract}
The methodological approach focuses on teaching and learning of the magnitude fifth grade area of basic primary education. It designs and implements a sequence of activities under the theory proposed by Brousseau teaching situations, and is structured according to the phases for the construction of the scale raised by Chamorro and Belmonte, which are mapped with Piagetian stages of development measuring thereby obtaining more detailed characterization to build any perceptible magnitude with the respective measurement unit in a progressive manner, in which are incorporated Rosa didactic knowledge Corberán which emphasize the magnitude treatment area and meet student needs.
\end{abstract}

Keywords: School math, measure, magnitude, area, design, management and evaluation, didactic.

1 Artículo de Investigación

2 Universidad Distrital Francisco José de Caldas. Bogotá, Colombia. Contacto: geraldstevengg@gmail.com

3 Universidad Distrital Francisco José de Caldas. Bogotá, Colombia. Contacto: yeimy.rodriguez.garcia@gmail.com 


\section{Resumo}

A abordagem metodológica incide sobre o ensino e aprendizagem de magnitude área da quinta série do ensino fundamental básico. Ela projeta e implementa uma seqüência de atividades com a teoria proposta por situações de ensino Brousseau, e está estruturado de acordo com as fases para a construção da escala criada por Chamorro e Belmonte, que são mapeados com os estágios de desenvolvimento de Piaget obtendo-se assim medir a caracterização mais detalhada para construir qualquer magnitude perceptível com a unidade de medição, de forma progressiva, em que são incorporados Rosa didáctico Corberán conhecimento que enfatizam a área de tratamento e a magnitude atender às necessidades dos alunos.

Palabras clave: Matemática do ensino médio, a medida, magnitude, área, design, gestão e avaliação didática.

\section{Contextualización}

La implementación de la propuesta didáctica se hace en la ciudad de Bogotá, en el colegio distrital Restrepo Millán, para el segundo periodo del 2012, con estudiantes de quinto grado de escolaridad. La experiencia de aula se centra en la enseñanza-aprendizaje de la magnitud área por medio de una situación fundamental que se encuentra implícita dentro de la secuencia de actividades, la cual se apoya en distintos materiales semióticos manipulativos para su desarrollo.

\section{Referentes teórico-prácticos básicos y metodología}

La secuencia didáctica se estructura teniendo en cuenta las fases para la construcción de la magnitud (percepción de una magnitud como propiedad que posee una colección de objetos, conservación y orden de la magnitud, establecimiento del número), según Chamorro \& Belmonte (1991); y se aplica la teoría de situaciones didácticas de Brousseau (1986), que se articula con la fase de evaluación propuesto por el grupo DECA (1992).

A las fases de construcción de la magnitud propuestas por Chamorro \& Belmonte (1991), se articulan los estadios piagetianos sobre el desarrollo de la medida (comparación perceptiva, desplazamiento de objetos, construcción de la unidad); y se hace una correspondencia con los dominios de conocimiento que Corberán (1996) propone, donde es más explícita la caracterización de la construcción de la magnitud área en cuanto a los conceptos que se deben abordar, y los procedimientos para su construcción.

Los diferentes materiales didácticos se clasifican según la propuesta de Godino (S.F) referente al uso de materiales Semióticos. Cabe aclarar que para el diseño de la actividad de diagnostico, se tienen en cuenta las dificultades para la construcción de la magnitud área identificadas por Zapata \& Cano (2008). Y se utiliza la definición formal de magnitud propuesta por Fiol \& Fortuny (1990), además de la definición dada por Godino, Batanero \& Roa (2002).

\section{Descripción general de la experiencia de aula}

La propuesta de enseñanza-aprendizaje, se justifica en la necesidad y posibilidad de que los alumnos de grado quinto superen las dificultades evidenciadas en la interpretación de magnitud área, tales como la confusión del área con el perímetro, y el desconocimiento de ambos conceptos como tal; dificultades que podrían conllevar a futuras necesidades mucho mas grandes como por ejemplo la manipulación de fórmulas y unidades estandarizadas de manera mecánica y por ende poco útiles ante situaciones reales según las afirmaciones de Corberán (1996), en concordancia con Zapata \& Cano (2008); razón por la cual se construyó el significado de superficie partiendo de una articulación teórica en la que se destacan las fases para la construcción de la magnitud, y los estadios piagetianos sobre el desarrollo 
de la medida propuestos por Chamorro y Belmonte (1991).

Para identificar el progreso de los estudiantes tras la aplicación de cada una de las actividades, se incluyó la propuesta didáctica de Corberán (1996), de la que se toman algunos elementos que sirvieron para abordar cada una de las fases de construcción, las cuales parten de la percepción del área y su diferenciación del perímetro de manera cualitativa, hasta el establecimiento de la unidad de superficie, su relación con el número, y la comprensión de su utilidad interfigural. Los recursos didácticos utilizados son semióticos de tipo verbal, textual, gráfico y tangible como preguntas orientadoras, planteamientos contextualizados en la realidad, dibujos, cintas de colores, fichas de tangram y unidades de teselación.

La secuencia se compone de 7 actividades, donde se encuentran incluidas la actividad de diagnóstico y la de evaluación; durante cada aplicación, se hizo uso de la teoría de situaciones didácticas de Brousseau (1986) lo que conllevo al desarrollo de una situación fundamental que estuvo implícita durante toda la secuencia; caracterización cualitativa y cuantitativa del área en relación con la realidad. Durante éste proceso se destacó el trabajo grupal y la construcción de cada uno de los conceptos implicados de manera constructivista debido a la manipulación de cada uno de los recursos didácticos hecha por los estudiantes de grado quinto del colegio Restrepo Millán.

\section{Logros y dificultades evidenciadas}

En este apartado se muestran las dificultades características de los estudiantes de quinto grado ante su primer encuentro con cada una de las actividades, $y$ los logros develados tras la ejecución de cada una de estas, para ello a continuación se hace mención a las fases de construcción de la magnitud donde estuvieron situadas estas actividades.

Percepción De La Magnitud: Inicialmente los alumnos no identifican con exactitud el área y el perímetro, razón por la cual se procedió con preguntas orientadoras ante la manipulación de materiales tangibles, que condujo a los alumnos a definir estos dos conceptos mediante una descripción de los mismos. Posteriormente de efectuar los diversos ejercicios de estimación directa, se evidencia que los alumnos construyeron el concepto de magnitud área como todo aquello que está dentro de una superficie delimitada, así como cada una de sus subregiones, lo cual dio base fundamental para el establecimiento del concepto de unidad de medida y su interiorización (Corberán, 1996). Se puede decir que gracias a los procedimientos de carácter geométrico los estudiantes finalmente no mostraron complicaciones para identificar el área en objetos de la realidad.

Conservación De La Magnitud: Al abordar esta fase los alumnos establecieron relaciones de orden, argumentadas en la forma que pudiera tener determinada figura, por ejemplo si la figura es más larga que otra, entonces "tiene mayor área" Corberán (1996), Zapata \& Cano (2008). Ante lo cual, la conservación de la magnitud se abordó con ejercicios de carácter geométrico y percepción visual, donde los educandos pudieron construir diferentes figuras con la misma área, dividida en subregiones (tangram), donde se valieron de las propiedades de congruencia y disección, para pasar de la estimación directa a la estimación analítica, y así concluir que sin importar la forma de las figuras, el área será la misma.

Abarcando otro aspecto de la conservación (el área como magnitud autónoma), cuando los alumnos determinaron una misma superficie mediante el uso de diferentes unidades de medida, no comprendían la razón por la cual las medidas eran diferentes; después de haber hecho un análisis de la descomposición de la superficie con respecto a las unidades, comprendieron que el área seguiría siendo la misma independientemente de la medida de estas unidades. Al establecer relaciones de orden o equivalencia respecto a una de las unidades, los estudiantes comprobaron de mejor manera que la dependencia del área respecto a la forma ya no era un argumento válido.

Orden De La Magnitud Y Relación Magnitud ÁreaNúmero: En cuanto a las relaciones de orden o equivalencia, se evidencian dificultades cuando los estudiantes pretendían comparar dos superficies diferentes; aquí se construyó la unidad mediante el desplazamiento de objetos (Chamorro \& Belmonte, 
1991); cuando se exponen las condiciones para qué una figura recubriera por completo una superficie (no pueden sobrar partes de la unidad, no pueden quedar espacios sin cubrir, no sobreponer figuras), los alumnos empiezan a comprender el área como las figuras que cubren por completo la superficie, de modo que cuando comprobaron la superficie dividida en subregiones, comprendieron que la unidad misma también se podía dividir (la unidad continua). Mediante la estimación analítica, los alumnos llegaron a la unidad como argumento principal para establecer estas relaciones de orden y equivalencia entre superficies haciendo su correspondencia con el número.

Finalmente se puede decir que aunque los alumnos hayan trabajado principalmente con figuras geométricas regulares, están familiarizados con una unidad situacional - figural y se encuentran en una transición entre el uso de procedimientos geométricos y numéricos bidimensionales para determinar áreas, existe la posibilidad de que tengan dificultades cuando se utilicen unidades irregulares debido a que la interfiguralidad de la unidad no se hizo explicita, sin embargo debido a las diferentes construcciones realizadas en esta secuencia, los alumnos poseen las herramientas necesarias para llegar a la unidad interfigural.

\section{Reflexión final}

En el proceso se cumplieron con sus objetivos, ya que los estudiantes construyeron la magnitud área como un concepto no sometido a fórmulas, sino a hechos que ellos mismos pudieron evidenciar, lo que implica que poseen los conocimientos requeridos para poder desarrollar a mayor profundidad el aspecto cuantitativo de ésta magnitud de manera significativa.

la magnitud área efectivamente es un concepto dependiente de muchas construcciones, que requieren de una percepción y análisis acerca de la misma en relación con la realidad; está ligada a procesos de comparación, en gran diversidad de situaciones, que desembocan en un tratamiento de carácter numérico caracterizado por ser cuantitativamente similar al tratamiento de otras magnitudes, lo cual implica que desde los procedimientos más simples hasta los más complejos, la construcción de la magnitud área da pie para construir otras magnitudes perceptibles, cosa que fue comprobado al aplicar lo establecido por Chamorro \& Belmonte (1991) respecto a las fases para la construcción de la magnitud a nivel general; es por eso que se considera que la realización de la secuencia didáctica dejo construcciones y herramientas importantes en los estudiantes de grado quinto de la institución educativa Restrepo Millán.

\section{Referencias}

Brousseau, G. (1986). Fondements et méthodes de la didactiques des mathématiques. Recherches en didactique des mathématiques, 7, 2, p. 33115. (Fundamentos y métodos de la didáctica de la matemática. Traducción de Dilma Fregona y Facundo Ortega). Argentina.

Chamorro, C. \& Belmonte, J. (1991). El problema de la medida: Didáctica De Las Magnitudes Lineales. (17).Madrid, España: Síntesis

Corberán, R. (1996). El área: Recursos Didácticos Para Su Enseñanza En Primaria. México

Fiol, L. \& Fortuny, J. (1990). Proporcionalidad directa: La Forma Y El Número. Madrid, España: Síntesis

Godino, J., Batanero, C., \& Roa, R. (2002). Medida de magnitudes y su didáctica para maestros. Recuperado de http://www.ugr.es/ jgodino/ edumat-maestros/manual/5_Medida.pdf

Godino, J. (S.F). Uso de material tangible y gráfico textual en el estudio de las matemáticas: Superando algunas posiciones ingenuas. Recuperado de http://princesacatherine. blogspot.com/2012/08/uso-de-material-tangible-y-grafico.html

Grupo DECA. (1992). Orientaciones para el diseño y elaboración de actividades de aprendizaje y de evaluación. Publicado en Revista Aula, No6, págs.: 33-39

Zapata, N. \& Cano, N. (2008). La enseñanza de la magnitud área. Universidad de Antioquia, 9 encuentro colombiano de Matemáticas educativas: ASOCOLME 